\title{
NONMONOTONEITY OF PICARD PRINCIPLE
}

\author{
BY
}

\author{
MITSURU NAKAI AND TOSHIMASA TADA ${ }^{1}$
}

Dedicated to Professor Tadashi Kuroda on the occasion of his 60 th birthday

\begin{abstract}
Two nonnegative $C^{\infty}$ functions $P(z)$ and $Q(z)$ on the punctured unit disk $0<|z| \leqslant 1$ are constructed such that $Q(z) \leqslant P(z)$ and there exists only one Martin minimal boundary point for the equation $\Delta u=P u$ over $z=0$ and, nevertheless, there exist exactly two Martin minimal boundary points for the equation $\Delta u=Q u$ over $z=0$.
\end{abstract}

The purpose of this paper is to show the existence of the following rather peculiar pair of two densities $P$ and $Q$ on the punctured unit disk $\Omega$ : $0<|z|<1$ with $0 \leqslant Q \leqslant P$ on $\Omega$ such that the Picard principle is valid for $P$ and invalid for $Q$ at the origin $z=0$.

We start fixing terminologies before explaining our result more precisely. We will take the punctured sphere $R: 0<|z| \leqslant \infty$ as our base Riemann surface so that every topological notion will be considered relative to $R=\{0<|z| \leqslant \infty\}$. Then the unit circle $|z|=1$ is the relative boundary $\partial \Omega$ of the punctured disk $\Omega: 0<|z|<1$ and the origin $z=0$ is the ideal boundary $\delta \Omega$ of $\Omega$.

By a density $P$ on $\Omega$ we mean a nonnegative locally Hölder continuous function $P(z)$ on the closure $\bar{\Omega}=\Omega \cup \partial \Omega$ so that $P$ may or may not have a singularity at $z=0$. With a density $P$ on $\Omega$ we associate the class $P P(\Omega ; \partial \Omega)$ of nonnegative real valued continuous functions $u$ on $\bar{\Omega}$ vanishing on $\partial \Omega$ such that $u$ satisfies the following selfadjoint elliptic equation of the second order:

$$
\Delta u(z)=P(z) u(z)
$$

on $\Omega$, where $\Delta$ is the Laplacian $4 \partial^{2} / \partial z \partial \bar{z}$. We also denote by $P P_{1}(\Omega ; \partial \Omega)$ the subclass of $P P(\Omega ; \partial \Omega)$ consisting of functions $u$ with the normalization

$$
-\int_{\partial \Omega} \frac{\partial}{\partial|z|} u(z)|d z|=2 \pi \text {. }
$$

The cardinal number \#(ex. $\left.P P_{1}(\Omega ; \partial \Omega)\right)$ of the set ex. $P P_{1}(\Omega ; \partial \Omega)$ of extreme points of the convex set $P P_{1}(\Omega ; \partial \Omega)$ is referred to as the Picard dimension of a density $P$ at the ideal boundary $\delta \Omega$ of $\Omega, \operatorname{dim} P$ in notation, i.e.

$$
\operatorname{dim} P=\#\left(\operatorname{ex.} P P_{1}(\Omega ; \partial \Omega)\right) .
$$

Received by the editors January 9, 1985.

1980 Mathematics Subject Classification. Primary 30F25; Seconary 31A10, 31A35.

${ }^{1}$ Both of the authors were supported in part by Grant-in-Aid for Scientific Research, No. 59340007, Japanese Ministry of Education, Science and Culture. 
There exists a bijective correspondence $u \leftrightarrow \mu$ between $P P_{1}(\Omega ; \partial \Omega)$ and the set of probability measures $\mu$ on ex. $P P_{1}(\Omega ; \partial \Omega)$ such that

$$
u=\int_{\text {ex. } P P_{1}(\Omega ; \partial \Omega)} v d \mu(v)
$$

as a consequence of the Choquet theorem (cf. e.g. [11]).

If $\operatorname{dim} P=1$, then we say that the Picard principle is valid for $P$. This means that $P P_{1}(\Omega ; \partial \Omega)$ consists of a single function $u_{0}$ so that for every nonnegative solution $h$ of (1) on $\Omega$ there exist a nonnegative real number $\lambda$ and a bounded solution $b$ of (1) on $0<|z|<r<1$ such that $h=\lambda u_{0}+b$. This property was first found by Picard in 1923 for the density $P \equiv 0$, i.e. for positive harmonic functions, with $u_{0}(z)=$ $-\log |z|$, which is also known as the principle of positive singularities. The above formulation, as being of Picard dimension one, is due to Bouligand in 1931 and the study of the Picard principle for general densities was initiated by Brelot [1, 2].

We are interested in characterizing all densities $P$ on $\Omega$ for which the Picard principle is valid. To a certain extent it is true that the Picard principle is valid for a density $P$ at $\delta \Omega$ if the singular behavior of $P$ at $\delta \Omega$ is not too wild. For example, if $P$ is in $L^{1}(\Omega)[8]$ or $P(z)=O\left(|z|^{-2}\right)$ as $z \rightarrow 0$ [4], then the Picard principle is valid for $P$. In view of these it may sound plausible that if the Picard principle is valid for a density $P$, then it is also valid for any density $Q \leqslant P$ since the singular behavior of $Q$ at $\delta \Omega$ must be less wild than that of $P$. This is certainly the case $[7,5]$ for densities $P$ and $Q$ which are rotation free, i.e. $P(z)=P(|z|)$ and $Q(z)=Q(|z|)$. The purpose of this paper is to show, contrary to this intuition, that the above expectation is not in general correct. Namely we will prove the following:

THE MAIN THEOREM. There exists a pair of densities $P$ and $Q$ on $\Omega$ such that $0 \leqslant Q \leqslant P$ on $\Omega$ and the Picard principle is valid for $P$ and invalid for $Q$.

The correspondence $P \mapsto \operatorname{dim} P$ is a mapping from the set $\mathscr{D}$ of densities on $\Omega$ into the set of cardinal numbers. We have seen $[9,10]$ that the range $\operatorname{dim} \mathscr{D}$ contains the set $\mathbf{N}$ of positive integers, the infinite countable cardinal number a and the cardinal number $c$ of the continuum so that $\operatorname{dim} \mathscr{D} \subset[1, c]$ in general and $\operatorname{dim} \mathscr{D}=$ $[1, \mathrm{c}]$ if the continuum hypothesis is postulated. We have asked [6] whether $P \rightarrow \operatorname{dim} P$ is monotone (i.e. whether $P \leqslant Q$ implies $\operatorname{dim} P \leqslant \operatorname{dim} Q$ ). The above result shows that it is not monotone in general on $\mathscr{D}$ although it is monotone [7] on $\mathscr{D}_{r}$, the set of rotation free densities.

The proof of the main theorem will be divided into three parts. In the first part, $\S 1$, two subregions of $\Omega$ with relative harmonic dimensions one and two, respectively, will be constructed. In $\$ 2$ we associate what we call firmly associated densities with the above two regions. In both of these two parts, the Schwarz alternating method, or the linear operator method for principal functions in the modern terminology of Rodin-Sario [12], will be intensively used. The proof will be completed in the final very short $\$ 3$.

\section{Relative harmonic dimensions.}

1. A sequence $\left\{\bar{Y}_{n}\right\}_{1}^{\infty}$ of closures $\bar{Y}_{n}$ of Jordan regions $Y_{n}$ in $\Omega$ will be referred to as a $\mathscr{Y}$-sequence in $\Omega$ if $\bar{Y}_{n} \cap \bar{Y}_{m}=\varnothing(n \neq m)$, so $W=W\left(\left\{\bar{Y}_{n}\right\}\right)=\Omega-\cup_{1}^{\infty} \bar{Y}_{n}$ is connected and $\left\{\bar{Y}_{n}\right\}$ converges to $\delta \Omega: z=0$, i.e. there exist only a finite number of 
$\bar{Y}_{n}$ such that $\bar{Y}_{n} \cap\{\varepsilon \leqslant|z|<1\} \neq \varnothing$ for any $\varepsilon>0$. We denote by $\partial D$ the relative boundary of a subregion $D$ of the punctured sphere $R=\{0<|z| \leqslant+\infty\}$ considered in $R$. We then consider the class $H P(W ; \partial W)$ of nonnegative harmonic functions on $W$ with vanishing boundary values on $\partial W$ and the subclass $H P_{1}(W ; \partial W)$ of $H P(W ; \partial W)$ consisting of those functions $u$ with the normalization (2). Similar to the Picard dimension we define the relative harmonic dimension, $\operatorname{dim}\left\{\bar{Y}_{n}\right\}$ in notation, of a $\mathscr{Y}$-sequence $\left\{\bar{Y}_{n}\right\}$ at $\delta \Omega: z=0$ by

$$
\operatorname{dim}\left\{\bar{Y}_{n}\right\}=\#\left(\text { ex. } H P_{1}(W ; \partial W)\right) .
$$

In subsection 6 we will give examples of $\mathscr{Y}_{\text {-sequences }}\left\{\bar{S}_{n}\right\}_{1}^{\infty}$ and $\left\{\bar{S}_{n j}\right\}_{j=1,2 ; n \geqslant 1}$ satisfying $\operatorname{dim}\left\{\bar{S}_{n}\right\}=1, \operatorname{dim}\left\{\bar{S}_{n j}\right\}=2$ and $\bigcup_{1}^{\infty}\left(\bar{S}_{n 1} \cup \bar{S}_{n 2}\right) \subset \bigcup_{1}^{\infty} \bar{S}_{n}$ after establishing auxiliary results in subsections $2-5$.

2. We fix positive nubmers $a, \delta, p, q$ and $\rho$ with $0<a-\delta<a+\delta<1,0<p-$ $\rho<p<q<q+\rho<2 \pi$ and consider a subregion (see Figure 1)

$$
\begin{aligned}
A= & A(b)=\{a-\delta<|z|<a+\delta, p-\rho<\arg z<p\} \\
& \cup\{a<|z|<b, p \leqslant \arg z \leqslant q\} \\
& \cup\{a-\delta<|z|<a+\delta, q<\arg z<q+\rho\}
\end{aligned}
$$

of $\Omega$ for any number $b$ with $a<b<a+\delta$. We also consider subsets $F^{-}, F^{+}$of $\partial A$ and subsets $\Gamma^{-}, \Gamma^{+}$of $A$ given by (see Figure 2)

$$
\begin{aligned}
& F^{-}=\{|z|=a-\delta, p-\rho<\arg z<p\} \\
& \cup\{a-\delta \leqslant|z| \leqslant a+\delta, \arg z=p-\rho\} \\
& \cup\{|z|=a+\delta, p-\rho<\arg z<p\}, \\
& F^{+}=\{|z|=a-\delta, q<\arg z<q+\rho\} \\
& \cup\{a-\delta \leqslant|z| \leqslant a+\delta, \arg z=q+\rho\} \\
& \cup\{|z|=a+\delta, q<\arg z<q+\rho\}, \\
& \Gamma^{-}=\Gamma^{-}(b)=\{a<|z|<b, \arg z=p\}, \\
& \Gamma^{+}=\Gamma^{+}(b)=\{a<|z|<b, \arg z=q\} .
\end{aligned}
$$

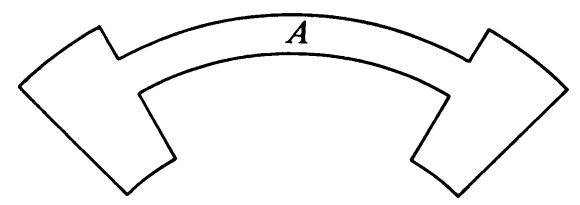

FIGURE 1
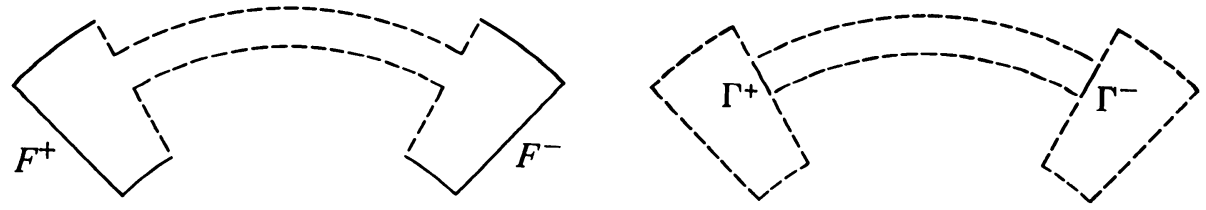

FigURE 2 




FIGURE 3

We denote by $\omega(\gamma ; D, \cdot)$ the harmonic measure of a subset $\gamma$ of $\partial D$ considered on a subregion $D$ of $R$. Then the family $\left\{u_{b}\right\}$ of harmonic measures

converges to 0 on $\Gamma^{-} \cup \Gamma^{+}$as $b \rightarrow a$ :

$$
u_{b}(z)=\omega\left(F^{-} \cup F^{+} ; A, z\right) \quad(z \in A)
$$

LEMMA $1 . \lim _{b \rightarrow a} \sup _{\Gamma^{-} \cup \Gamma^{+}} u_{b}=0$.

3. We prove Lemma 1. The harmonic measure $u_{b}$ dominates the harmonic measure

$$
w^{-}(z)=\omega\left(F^{-} ; A \cap\{p-\rho<\arg z<p\}, z\right)
$$

and is dominated by the harmonic measure

$$
v_{b}^{-}(z)=\omega\left(F^{-} \cup \Gamma^{-} ; A \cap\{p-\rho<\arg z<p\}, z\right) .
$$

Fix any positive number $\varepsilon$. Then there exist positive numbers $\delta_{0}, \rho_{0}$ such that $\delta_{0}<\delta, \rho_{0}<\rho$ and $w^{-}<\varepsilon$ on (see Figure 3)

$$
\begin{aligned}
F_{0}^{-}= & \left\{|z|=a-\delta_{0}, p-\rho_{0}<\arg z<p\right\} \\
& \cup\left\{a-\delta_{0} \leqslant|z| \leqslant a+\delta_{0}, \arg z=p-\rho_{0}\right\} \\
& \cup\left\{|z|=a+\delta_{0}, p-\rho_{0}<\arg z<p\right\} .
\end{aligned}
$$

The family $\left\{v_{b}^{-}\right\}$converges decreasingly to $w^{-}$as $b \rightarrow a$ and hence by the Dini theorem $v_{b}^{-}$converges uniformly on every compact subset of

$$
\bar{A} \cap\{p-\rho \leqslant \arg z \leqslant p\}-\left\{(a-\delta) e^{i p}, a e^{i p},(a+\delta) e^{i p}\right\} .
$$

Therefore there exists $b_{0}=b_{0}(\varepsilon)$ with $a<b_{0}<a+\delta_{0}$ such that $v_{b}^{-}-w^{-}<\varepsilon$ on $F_{0}^{-}$ for every $b$ with $a<b \leqslant b_{0}$. Then we have $u_{b}<v_{b}^{-}<2 \varepsilon$ on $F_{0}^{-}$and similarly $u_{b}<v_{b}^{+}<2 \varepsilon$ on

$$
\begin{aligned}
F_{0}^{+}= & \left\{|z|=a-\delta_{0}, q<\arg z<q+\rho_{0}\right\} \\
& \cup\left\{a-\delta_{0} \leqslant|z| \leqslant a+\delta_{0}, \arg z=q+\rho_{0}\right\} \\
& \cup\left\{|z|=a+\delta_{0}, q<\arg z<q+\rho_{0}\right\},
\end{aligned}
$$

where

$$
v_{b}^{+}(z)=\omega\left(F^{+} \cup \Gamma^{+} ; A \cap\{q<\arg z<q+\rho\}, z\right) .
$$

By the maximum principle, $u_{b}<2 \varepsilon$ is valid on a region (see Figure 4)

$$
\begin{aligned}
\left\{a-\delta_{0}\right. & \left.<|z|<a+\delta_{0}, p-\rho_{0}<\arg z<p\right\} \\
\cup & \{a<|z|<b, p \leqslant \arg z \leqslant q\} \\
\cup & \left\{a-\delta_{0}<|z|<a+\delta_{0}, q<\arg z<q+\rho_{0}\right\}
\end{aligned}
$$

including $\Gamma^{-} \cup \Gamma^{+}$. 


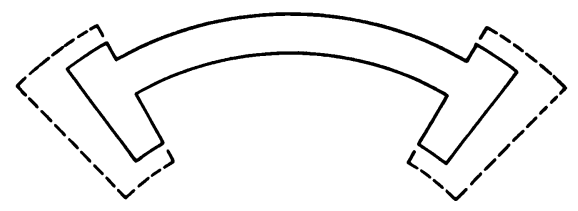

Figure 4

4. Let $D$ be a subregion of $R, \gamma$ a subset of $\partial D$ consisting of a finite number of disjoint analytic arcs such that $\overline{\partial D-\gamma} \cap(\gamma-e(\gamma))=\varnothing$ for the set $e(\gamma)$ of end points of each arc in $\gamma$. The Harnack constant $k=k(F)$ of a closed subset $F$ of $D$ such that $\bar{F}$ is compact and contained in $D \cup(\gamma-e(\gamma))$ is given by

$$
\begin{aligned}
k & =k(F)=k(F ; D, \gamma, a) \\
& =\sup \left\{\frac{u(z)}{u(a)} ; z \in F, u \in H P(D ; \gamma)-\{0\}\right\},
\end{aligned}
$$

where $a \in D$ and $H P(D ; \gamma)$ is the set of nonnegative harmonic functions on $D$ with vanishing boundary values on $\gamma$. Then we have the following

LEMMA 2. $k(F)<\infty$.

This is a special case of the so-called Carleson lemma but in our case of two dimension the existence of the conformal mapping reduces the lemma to a simple estimation of the Poisson kernel, which we describe in the sequel for the sake of completeness.

5. We assume that $F$ contains $a$ since $k(F) \leqslant k(F \cup\{a\})$. Let $\gamma_{1}, \ldots, \gamma_{s}$ be disjoint analytic arcs with $\gamma=\gamma_{1} \cup \cdots \cup \gamma_{s}$. Then we divide $F$ into a compact subset $F_{0}$ of $D$ and closed subsets $F_{1}, \ldots, F_{s}$ of $D$ satisfying $F=F_{0} \cup F_{1} \cup \cdots \cup F_{s}$, $a \in F_{0} \cap F_{1} \cap \cdots \cap F_{s}, \bar{F}_{j} \subset D \cup\left(\gamma_{j}-e\left(\gamma_{j}\right)\right)(j=1, \ldots, s)$, and $F_{j}$ is contained in a simply connected subregion of $R$. Further we take an analytic arc $\Gamma_{j}$ such that $\Gamma_{j} \cup \gamma_{j}$ encloses a simply connected subregion $D_{j}$ of $D$ containing $F_{j}$. Observe the facts that $k(F) \leqslant \max _{0 \leqslant j \leqslant s} k\left(F_{j}\right), k\left(F_{0}\right)<\infty, k\left(F_{j}\right) \leqslant k\left(F_{j} ; D_{j}, \gamma_{j}, a\right)$, and $D_{j}$ may be viewed as the unit disk by using a conformal mapping. Now it is sufficient to prove Lemma 2 in the case that $D=\{|z|<1\}, \gamma$ is a single circular arc, $a=0$ and $a$ is in $F$.

Let $u$ be any positive function in $H P(D ; \gamma)$ and $\mu$ be the Poisson-Herglotz (Martin) representing measure of $u$ :

$$
u(z)=\frac{1}{2 \pi} \int_{0}^{2 \pi} \operatorname{Re}\left(\frac{\zeta+z}{\zeta-z}\right) d \mu(\zeta) .
$$

The support of $\mu$ is contained in $\Gamma=\{|z|=1\}-(\gamma-e(\gamma))$ which has a positive distance $d$ from $F$. Then the Poisson kernel satisfies that

$$
\operatorname{Re}\left(\frac{\zeta+z}{\zeta-z}\right) \leqslant \frac{|\zeta+z|}{|\zeta-z|} \leqslant \frac{2}{d} \quad(\zeta \in \Gamma, z \in F) .
$$


Therefore we have

$$
u(z) \leqslant \frac{1}{2 \pi} \int_{\Gamma} \frac{2}{d} d \mu(\zeta)=\frac{2}{d} u(0) \quad(z \in F)
$$

so that $k(F) \leqslant 2 / d$.

6. In this subsection we give two $\mathscr{Y}$-sequences $\left\{\bar{S}_{n}\right\}_{1}^{\infty}$ and $\left\{\bar{S}_{n j}\right\}_{j=1,2 ; n \geqslant 1}$ with $\cup_{1}^{\infty}\left(\bar{S}_{n 1} \cup \bar{S}_{n 2}\right) \subset \cup_{1}^{\infty} \bar{S}_{n}$ and prove in subsections $7-9$ that $\operatorname{dim}\left\{\bar{S}_{n}\right\}=1, \operatorname{dim}\left\{\bar{S}_{n j}\right\}$ $=2$. Fix a sequence $\left\{a_{n}\right\}_{1}^{\infty}$ in $(0,1)$ with $a_{n}>a_{n+1}$ and $\lim _{n} a_{n}=0$. Fix positive numbers $p_{1}, q_{1}, p_{2}, q_{2}$ with $0<p_{1}<q_{1}<p_{2}<q_{2}<2 \pi$. Then we consider $\mathscr{Y}$ sequences $\left\{\bar{S}_{n}\right\}_{1}^{\infty},\left\{\bar{S}_{n j}\right\}_{j=1,2 ; n \geqslant 1}$ for any sequence $\left\{b_{n}\right\}_{1}^{\infty}$ in $(0,1)$ with $a_{n}>b_{n}>$ $a_{n+1}$ given by (see Figure 5)

$$
\begin{gathered}
S_{n}=S_{n}\left(\left\{b_{n}\right\}\right)=\left\{b_{n}<|z|<a_{n}, p_{1}<\arg z<q_{2}\right\}, \\
S_{n j}=S_{n j}\left(\left\{b_{n}\right\}\right)=\left\{b_{n}<|z|<a_{n}, p_{j}<\arg z<q_{j}\right\} \quad(j=1,2 ; n=1,2, \ldots) .
\end{gathered}
$$

We also consider subregions (see Figures 6 and 7)

$$
\begin{aligned}
& \Omega_{1}=\Omega-\left\{0<|z| \leqslant a_{1}, p_{1} \leqslant \arg z \leqslant q_{2}\right\}, \\
& \Omega_{2}=\Omega-\bigcup_{j=1}^{2}\left\{0<|z| \leqslant a_{1}, p_{j} \leqslant \arg z \leqslant q_{j}\right\}, \\
& W_{1}=W_{1}\left(\left\{b_{n}\right\}\right)=W\left(\left\{\bar{S}_{n}\right\}\right)=\Omega-\bigcup_{n=1}^{\infty} \bar{S}_{n}, \\
& W_{2}=W_{2}\left(\left\{b_{n}\right\}\right)=W\left(\left\{\bar{S}_{n j}\right\}\right)=\Omega-\bigcup_{n=1}^{\infty}\left(\bar{S}_{n 1} \cup \bar{S}_{n 2}\right)
\end{aligned}
$$

and mappings

$$
T_{\nu} u=u-H_{u}^{\Omega_{\nu}} \quad\left(u \in H P\left(W_{\nu} ; \partial W_{\nu}\right) ; \nu=1,2\right)
$$

of $H P\left(W_{\nu} ; \partial W_{\nu}\right)$ to $H P\left(\Omega_{\nu} ; \partial \Omega_{\nu}\right)$, where $H_{f}^{D}$ is defined as follows (cf. e.g. [3]). Let $D$ be a nonvoid open subset of $R$ not necessarily relatively compact such that each point of $\partial D$ is contained in a nondegenerate continuum contained in $\partial D$. Let $f$ be a continuous function on $\partial D$ such that there exists a nonnegative superharmonic function $s$ on $D$ whose lower limit boundary values exceed $|f|$ on $\partial D$. Firstly, in case $f \geqslant 0$ on $\partial D$, we define $H_{f}^{D}$ to be the lower envelope of the family of nonnegative superharmonic functions $s$ on $D$ with lower limit boundary values of $s \geqslant f$ on $\partial D$. For a general $f$ on $\partial D$ we define

$$
H_{f}^{D}=H_{\max (f, 0)}^{D}-H_{\max (-f, 0)}^{D} .
$$

Then $H_{f}^{D}$ is harmonic on $D$ and has boundary values $f$ on $\partial D$. In case $f \geqslant 0, H_{f}^{D}$ is the least nonnegative harmonic function on $D$ with boundary values $f$ on $\partial D$. It is easy to see that the mappings $T_{\nu}$ are order preserving (i.e. $u_{1} \leqslant u_{2}$ implies $T_{\nu} u_{1} \leqslant$ $T_{\nu} u_{2}$ ), positively homogeneous (i.e. $T_{\nu}(\lambda u)=\lambda T_{\nu} u$ for nonnegative numbers $\lambda$ ), and additive (i.e. $\left.T_{\nu}\left(u_{1}+u_{2}\right)=T_{\nu} u_{1}+T_{\nu} u_{2}\right)$. If we choose the sequence $\left\{b_{n}\right\}$ so as to make the sequence $\left\{b_{n}-a_{n+1}\right\}_{1}^{\infty}$ converge to zero rapidly enough, as described in the sequel, then we can show that the mappings $T_{1}, T_{2}$ are bijective. 

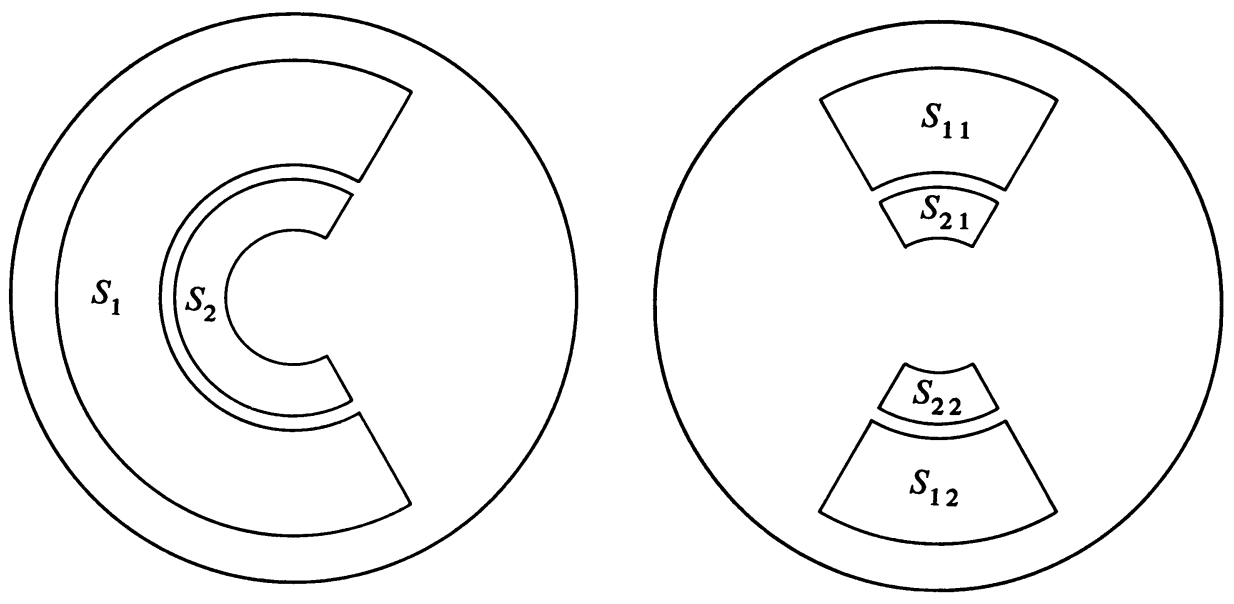

Figure 5
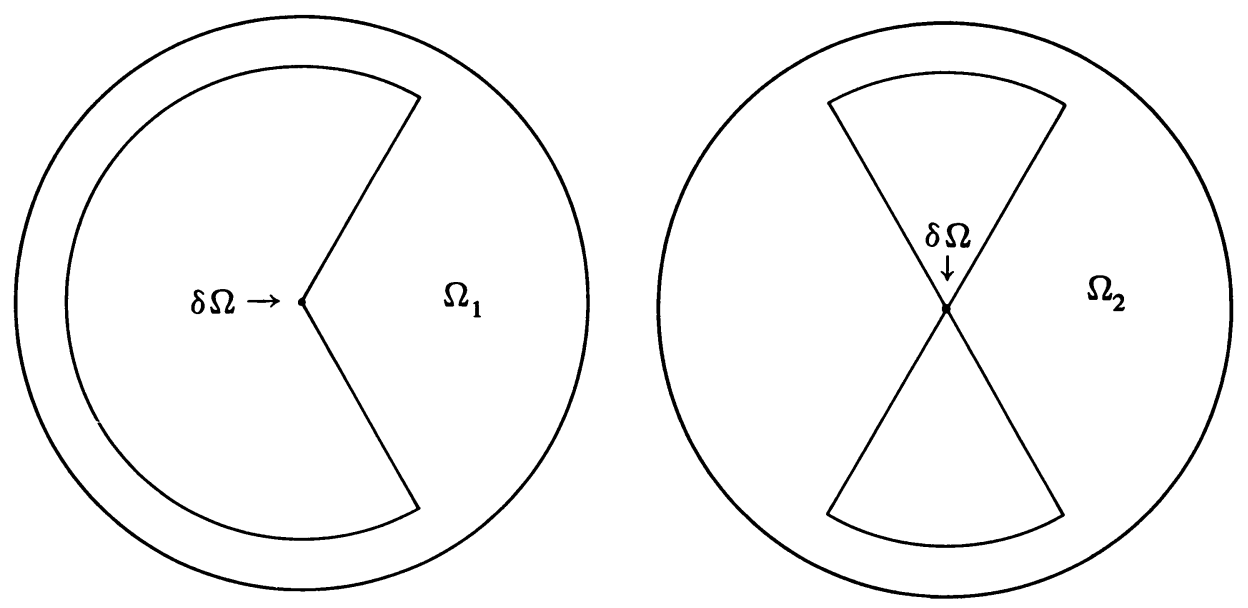

Figure 6
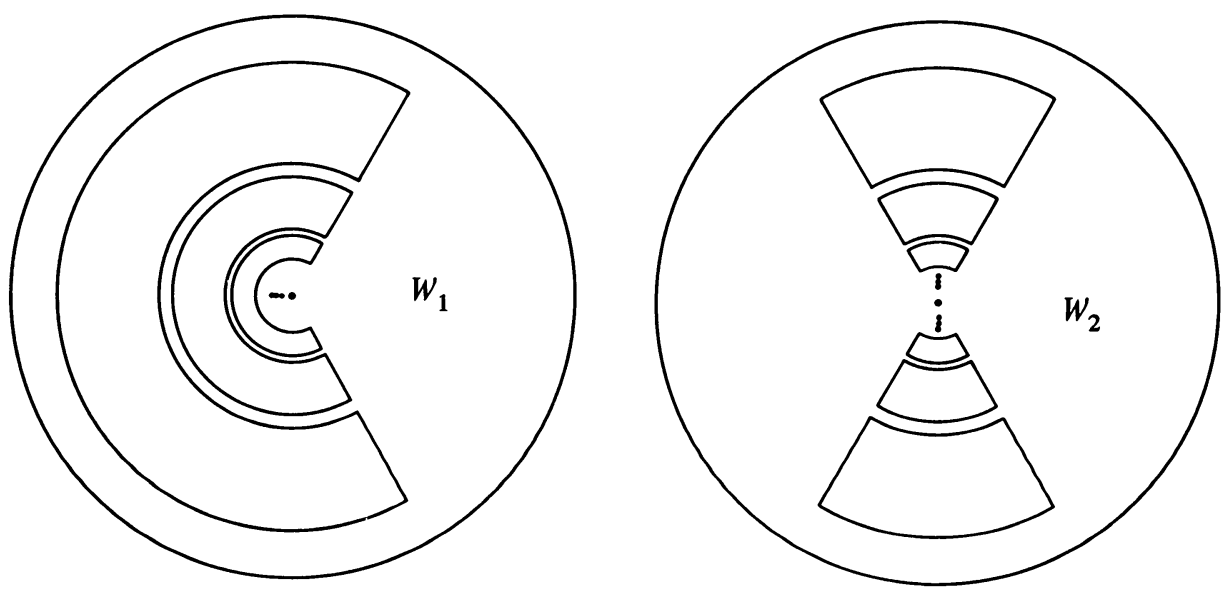

FIGURE 7 

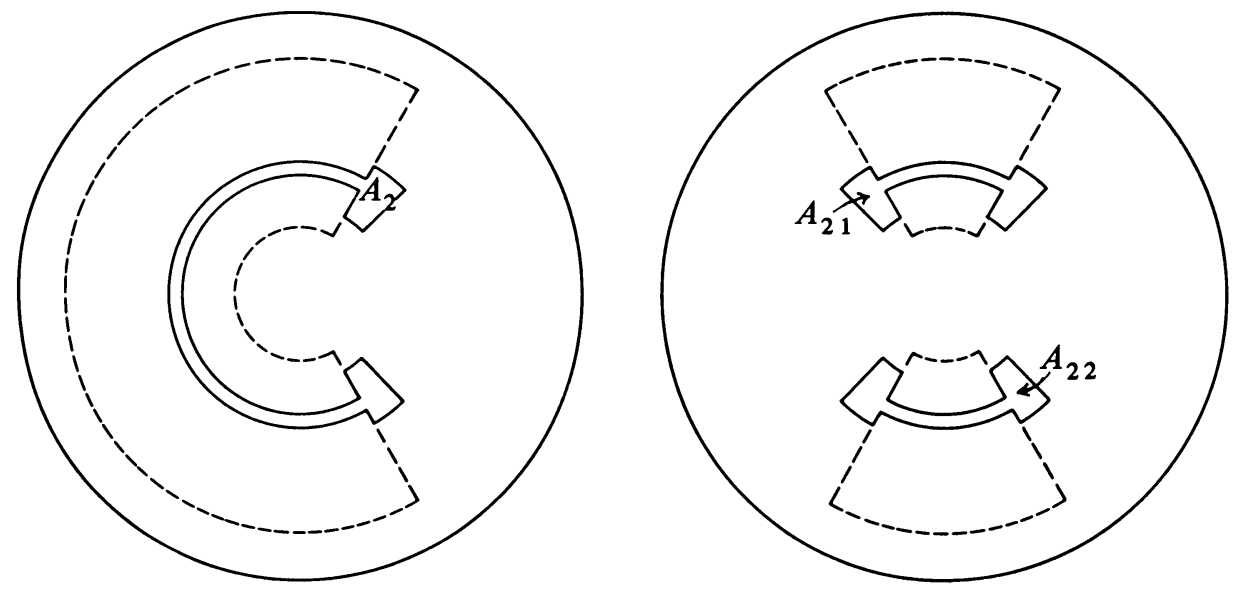

FIGURE 8

We fix a positive number $\rho$ with

$$
\rho<\min \left(p_{1},\left(p_{2}-q_{1}\right) / 2,2 \pi-q_{2}\right)
$$

and a sequence $\left\{\delta_{n}\right\}_{2}^{\infty}$ of positive numbers $\delta_{n}$ with

$$
\delta_{n}<\min \left(\left(a_{n-1}-a_{n}\right) / 2,\left(a_{n}-a_{n+1}\right) / 2\right) .
$$

Then the first property which $\left\{b_{n}\right\}$ has to satisfy is

$$
b_{n}<a_{n+1}+\delta_{n+1} / 2 \quad(n=1,2, \ldots) .
$$

For the sequence $\left\{b_{n}\right\}$ with this property we consider subregions (see Figure 8)

$$
\begin{aligned}
A_{n} & =A_{n}\left(\left\{b_{n}\right\}\right) \\
& =W_{1} \cap\left\{a_{n}-\delta_{n}<|z|<a_{n}+\delta_{n}, p_{1}-\rho<\arg z<q_{2}+\rho\right\}, \\
A_{n j} & =A_{n j}\left(\left\{b_{n}\right\}\right) \\
& =W_{2} \cap\left\{a_{n}-\delta_{n}<|z|<a_{n}+\delta_{n}, p_{j}-\rho<\arg z<q_{j}+\rho\right\} \\
& \quad(j=1,2 ; n=2,3, \ldots)
\end{aligned}
$$

of $W_{1}, W_{2}$, respectively and closed subsets (see Figure 9)

$$
\begin{aligned}
& F_{n}^{-}=\partial A_{n} \cap\left\{p_{1}-\rho \leqslant \arg z<p_{1}\right\}, \\
& F_{n}^{+}=\partial A_{n} \cap\left\{q_{2}<\arg z \leqslant q_{2}+\rho\right\}, \\
& F_{n j}^{-}=\partial A_{n j} \cap\left\{p_{j}-\rho \leqslant \arg z<p_{j}\right\}, \\
& F_{n j}^{+}=\partial A_{n j} \cap\left\{q_{j}<\arg z \leqslant q_{j}+\rho\right\} \quad(j=1,2 ; n=2,3, \ldots)
\end{aligned}
$$

of $\Omega_{1}$ or $\Omega_{2}$.

We also consider closed subsets (see Figure 10)

$$
\begin{aligned}
& \Gamma_{n}^{-}=\Gamma_{n}^{-}\left(\left\{b_{n}\right\}\right)=\left\{a_{n}<|z|<b_{n-1}, \arg z=p_{1}\right\}, \\
& \Gamma_{n}^{+}=\Gamma_{n}^{+}\left(\left\{b_{n}\right\}\right)=\left\{a_{n}<|z|<b_{n-1}, \arg z=q_{2}\right\}, \\
& \Gamma_{n j}^{-}=\Gamma_{n j}^{-}\left(\left\{b_{n}\right\}\right)=\left\{a_{n}<|z|<b_{n-1}, \arg z=p_{j}\right\}, \\
& \Gamma_{n j}^{+}=\Gamma_{n j}^{+}\left(\left\{b_{n}\right\}\right)=\left\{a_{n}<|z|<b_{n-1}, \arg z=q_{j}\right\}
\end{aligned}
$$


of $A_{n}$ or $A_{n j}$ and subsets (see Figure 11)

$$
\begin{aligned}
\gamma_{n}^{-}= & \left\{a_{n}-5 \delta_{n} / 4 \leqslant|z| \leqslant a_{n}-3 \delta_{n} / 4, \arg z=p_{1}\right\} \\
& \cup\left\{a_{n}+3 \delta_{n} / 4 \leqslant|z| \leqslant a_{n}+5 \delta_{n} / 4, \arg z=p_{1}\right\}, \\
\gamma_{n}^{+}= & \left\{a_{n}-5 \delta_{n} / 4 \leqslant|z| \leqslant a_{n}-3 \delta_{n} / 4, \arg z=q_{2}\right\} \\
& \cup\left\{a_{n}+3 \delta_{n} / 4 \leqslant|z| \leqslant a_{n}+5 \delta_{n} / 4, \arg z=q_{2}\right\}, \\
\gamma_{n j}^{-}= & \left\{a_{n}-5 \delta_{n} / 4 \leqslant|z| \leqslant a_{n}-3 \delta_{n} / 4, \arg z=p_{j}\right\} \\
& \cup\left\{a_{n}+3 \delta_{n} / 4 \leqslant|z| \leqslant a_{n}+5 \delta_{n} / 4, \arg z=p_{j}\right\}, \\
\gamma_{n j}^{+}= & \left\{a_{n}-5 \delta_{n} / 4 \leqslant|z| \leqslant a_{n}-3 \delta_{n} / 4, \arg z=q_{j}\right\} \\
& \cup\left\{a_{n}+3 \delta_{n} / 4 \leqslant|z| \leqslant a_{n}+5 \delta_{n} / 4, \arg z=q_{j}\right\} \\
& \quad(j=1,2 ; n=2,3, \ldots)
\end{aligned}
$$

of $\partial \Omega_{1}$ or $\partial \Omega_{2}$.
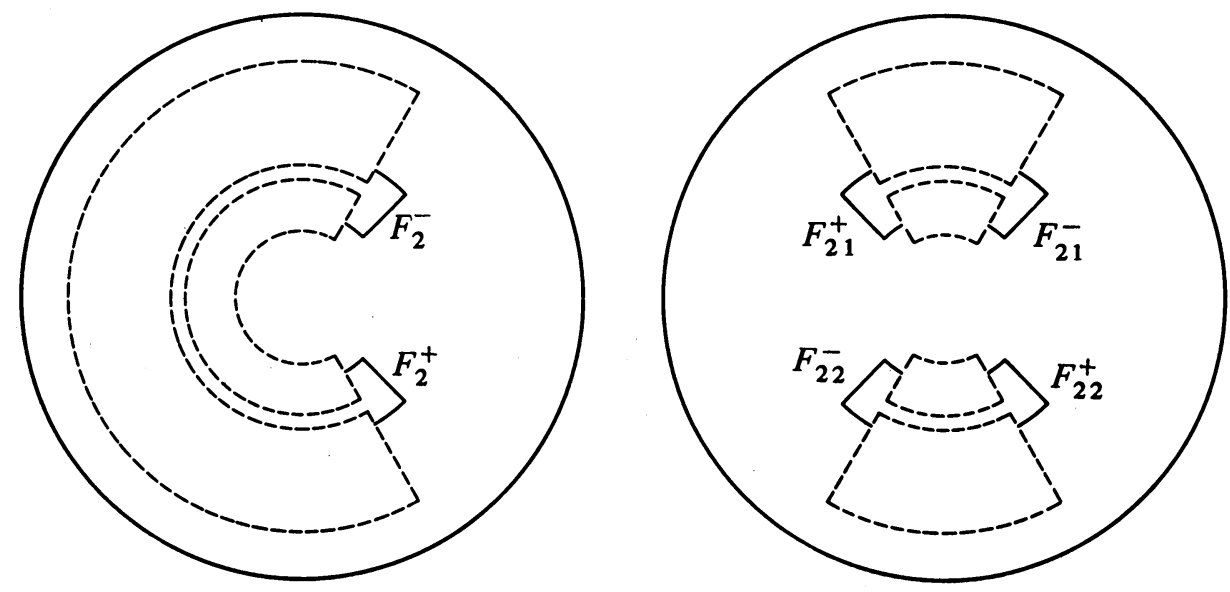

FigURE 9
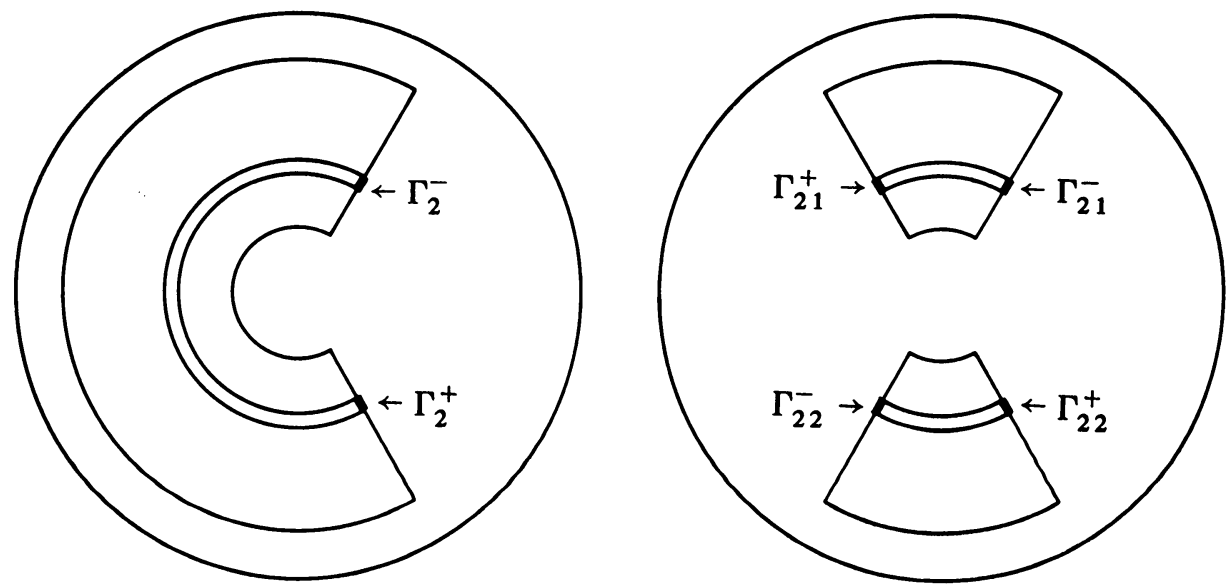

Figure 10 

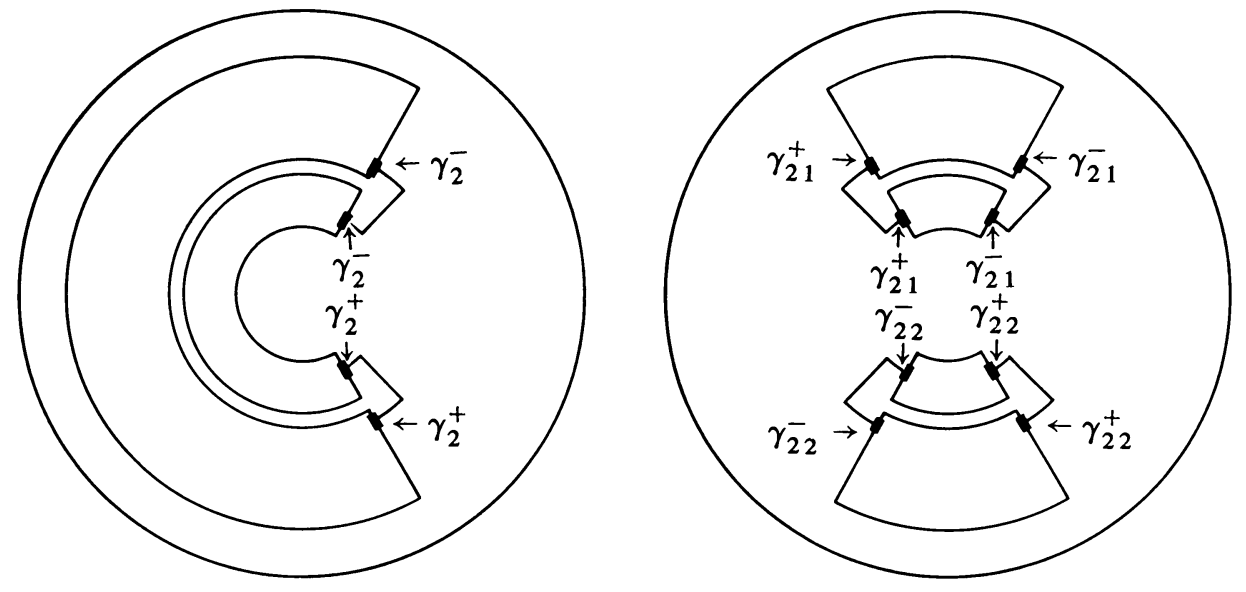

FigURE 11

Then Harnack constants

$$
\begin{aligned}
& k_{n}^{-}=k\left(F_{n}^{-} ; \Omega_{1}, \gamma_{n}^{-}, 1 / 2\right), \quad k_{n}^{+}=k\left(F_{n}^{+} ; \Omega_{1}, \gamma_{n}^{+}, 1 / 2\right), \\
& k_{n j}^{-}=k\left(F_{n j}^{-} ; \Omega_{2}, \gamma_{n j}^{-}, 1 / 2\right), \quad k_{n j}^{+}=k\left(F_{n j}^{+} ; \Omega_{2}, \gamma_{n j}^{+}, 1 / 2\right)
\end{aligned}
$$

are finite by Lemma 2 and independent of $\left\{b_{n}\right\}$. Set

$$
k_{n}=\max \left(k_{n}^{-}, k_{n}^{+}, k_{n 1}^{-}, k_{n 1}^{+}, k_{n 2}^{-}, k_{n 2}^{+}\right) .
$$

In view of Lemma 1 we can choose the sequence $\left\{b_{n}\right\}$ so as to satisfy

$$
\begin{cases}\omega\left(F_{n}^{-} \cup F_{n}^{+} ; A_{n}, z\right) \leqslant 1 / 2 k_{n} & \left(z \in \Gamma_{n}^{-} \cup \Gamma_{n}^{+}\right), \\ \omega\left(F_{n j}^{-} \cup F_{n j}^{+} ; A_{n j}, z\right) \leqslant 1 / 2 k_{n} & \left(z \in \Gamma_{n j}^{-} \cup \Gamma_{n j}^{+}\right)\end{cases}
$$

for every $j=1,2$ and $n=2,3, \ldots$. Now the second property which $\left\{b_{n}\right\}$ has to satisfy is (5). Then we will prove in subsections $7-8$ the following

THEOREM 1. If the sequence $\left\{b_{n}\right\}_{1}^{\infty}$ satisfies (4) and (5), then the mappings $T_{1}$ and $T_{2}$ are bijective.

7. First we prove that $T_{2}$ is injective. The similar argument will also prove the injectivity of $T_{1}$. Let $u_{1}, u_{2}$ be any functions in $H P\left(W_{2} ; \partial W_{2}\right)$. Then $u_{1}, u_{2}$ satisfy

$$
u_{1} \leqslant k_{n} u_{1}(1 / 2), \quad u_{2} \leqslant k_{n} u_{2}(1 / 2)
$$

on $F_{n j}^{-} \cup F_{n j}^{+}(j=1,2 ; n=2,3, \ldots)$. Therefore, by (5), $u_{1}, u_{2}$ satisfy

$$
u_{1} \leqslant u_{1}(1 / 2) / 2, \quad u_{2} \leqslant u_{2}(1 / 2) / 2
$$

on $\Gamma_{2}=\bigcup_{n=2}^{\infty} \cup_{j=1}^{2}\left(\Gamma_{n j}^{-} \cup \Gamma_{n j}^{+}\right)$. On the other hand $u_{1}, u_{2}$ vanish on $\partial \Omega_{2}-\Gamma_{2}$ so that $u_{1}-u_{2}$ is bounded on $\partial \Omega_{2}$. Assume $T_{2} u_{1}=T_{2} u_{2}$. Since $u_{1}-u_{2}=H_{u_{1}-u_{2}}^{\Omega_{2}}$ on $\Omega_{2}, u_{1}-u_{2}$ is bounded on $\Omega_{2}$ and hence on $W_{2}$. Further $u_{1}-u_{2}$ vanishes on $\partial W_{2}$. Then we have $u_{1}-u_{2} \equiv 0$. 
8. Next we prove that $T_{2}$ is surjective. The similar argument will also prove the surjectivity of $T_{1}$. We set

$$
F_{2}=\bigcup_{n=2}^{\infty} \bigcup_{j=1}^{2}\left(F_{n j}^{-} \cup F_{n j}^{+}\right), \quad \Gamma_{2}=\bigcup_{n=2}^{\infty} \bigcup_{j=1}^{2}\left(\Gamma_{n j}^{-} \cup \Gamma_{n j}^{+}\right) .
$$

Consider a bounded operator $K_{2}$ of $C\left(F_{2}\right)$ to $C\left(\Gamma_{2}\right)$ defined by

$$
K_{2} \phi(z)=H_{\phi}^{A_{n j}}(z) \quad\left(\phi \in C\left(F_{2}\right), z \in \Gamma_{n j}^{-} \cup \Gamma_{n j}^{+} ; j=1,2 ; n=2,3, \ldots\right),
$$

where $C(E)$ denotes the set of continuous functions on a subset $E$ of $R$ and for each $\phi \in C\left(F_{2}\right)$ we define $\phi=0$ on $\bigcup_{2}^{\infty}\left(\partial A_{n 1} \cup \partial A_{n 2}\right)-F_{2}$. Also consider a bounded operator $L_{2}$ of $C B\left(\Gamma_{2}\right)$ to $C B\left(F_{2}\right)$ defined by

$$
L_{2} \psi(z)=H_{\psi}^{\Omega_{2}}(z) \quad\left(\psi \in C B\left(\Gamma_{2}\right), z \in F_{2}\right),
$$

where $C B(E)$ denotes the set of bounded continuous functions on a subset $E$ of $R$ and for each $\psi \in C B\left(\Gamma_{2}\right)$ we define $\psi=0$ on $\partial \Omega_{2}-\Gamma_{2}$. Let $v$ be any function in $H P\left(\Omega_{2} ; \partial \Omega_{2}\right)$. Thus we can construct a function $u$ in $H P\left(W_{2} ; \partial W_{2}\right)$ with $T_{2} u=v$ by using the bounded operator $M_{2}=L_{2} \circ K_{2}$ of a subspace of $C\left(F_{2}\right)$ to $C B\left(F_{2}\right)$. Since $v$ is dominated by the constant $k_{n} v(1 / 2)$ on $\bigcup_{j=1}^{2}\left(F_{n j}^{-} \cup F_{n j}^{+}\right)(n=2,3, \ldots)$, we have, by (5), $K_{2} v \leqslant v(1 / 2) / 2$ on $\Gamma_{2}$. Then the harmonic function $M_{2} v$ on $\Omega_{2}$ is dominated by the constant $v(1 / 2) / 2$ and hence by induction $M_{2}^{m} v$ is dominated by the constant $v(1 / 2) / 2^{m}(m=1,2, \ldots)$. Therefore there exists a nonnegative function $\phi_{v}=\sum_{m=0}^{\infty} M_{2}^{m} v$ on $F_{2}$ with $\left(I-M_{2}\right) \phi_{v}=v$, where $I$ is the identity operator of $C\left(F_{2}\right)$. Observe that $K_{2} \phi_{v}$ is a harmonic function on $A_{2}=\cup_{2}^{\infty}\left(A_{n 1} \cup A_{n 2}\right)$ and $v+M_{2} \phi_{v}$ is a harmonic function on $\Omega_{2}$ with the same values as that of $K_{2} \phi_{v}$ on $\partial\left(\Omega_{2} \cap A_{2}\right)$. Then we can define the harmonic function

$$
u(z)= \begin{cases}K_{2} \phi_{v}(z) & \left(z \in A_{2}\right), \\ v(z)+M_{2} \phi_{v}(z) & \left(z \in \Omega_{2}\right)\end{cases}
$$

in $H P\left(W_{2} ; \partial W_{2}\right)$ which satisfies on $\Omega_{2}$

$$
T_{2} u=u-H_{u}^{\Omega_{2}}=v+M_{2} \phi_{v}-L_{2}\left(K_{2} \phi_{v}\right)=v .
$$

9. We prove $\operatorname{dim}\left\{\bar{S}_{n}\right\}=1, \operatorname{dim}\left\{\bar{S}_{n j}\right\}=2$. Consider a mapping $E_{\nu}$ of $H P_{1}\left(W_{\nu} ; \partial W_{\nu}\right)$ to $H P_{1}\left(\Omega_{\nu} ; \partial \Omega_{\nu}\right)$ given by

$$
E_{\nu} u=\frac{T_{\nu} u}{l\left(T_{\nu} u\right)} \quad\left(u \in H P_{1}\left(W_{\nu} ; \partial W_{\nu}\right) ; \nu=1,2\right),
$$

where $l(v)=-(2 \pi)^{-1} \int_{\partial \Omega}(\partial / \partial|z|) v(z)|d z|$. Let $u$ be in ex. $H P_{1}\left(W_{\nu} ; \partial W_{\nu}\right)$ and $E_{\nu} u$ be represented in a form $E_{\nu} u=t v_{1}+(1-t) v_{2}$ for a constant $t$ in $(0,1)$ and $v_{1}, v_{2}$ in $H P_{1}\left(\Omega_{\nu} ; \partial \Omega_{\nu}\right)$. Then we have

$$
\frac{u}{l\left(T_{\nu} u\right)}=t l\left(T_{\nu}^{-1} v_{1}\right) \frac{T_{\nu}^{-1} v_{1}}{l\left(T_{\nu}^{-1} v_{1}\right)}+(1-t) l\left(T_{\nu}^{-1} v_{2}\right) \frac{T_{\nu}^{-1} v_{2}}{l\left(T_{\nu}^{-1} v_{2}\right)}
$$

Therefore we have $u=T^{-1} v_{1} / l\left(T_{\nu}^{-1} v_{1}\right)=T^{-1} v_{2} / l\left(T_{\nu}^{-1} v_{2}\right)$ and hence $l\left(T_{\nu} u\right) E_{\nu} u=$ $v_{1} / l\left(T_{\nu}^{-1} v_{1}\right)=v_{2} / l\left(T_{\nu}^{-1} v_{2}\right)$. Then $l\left(E_{\nu} u\right)=l v_{1}=l v_{2}=1$ imply $E_{\nu} u=v_{1}=v_{2}$ so that $E_{\nu} u$ is extreme in $H P_{1}\left(\Omega_{\nu} ; \partial \Omega_{\nu}\right)$. Thus $E_{\nu}$ is a mapping of ex. $H P_{1}\left(W_{\nu} ; \partial W_{\nu}\right)$ to 
ex. $H P_{1}\left(\Omega_{\nu} ; \partial \Omega_{\nu}\right)$. Further we can show that $E_{\nu}$ is bijective. Let $u_{1}, u_{2}$ be any functions in ex. $H P_{1}\left(W_{\nu} ; \partial W_{\nu}\right)$ such that $E_{\nu} u_{1}=E_{\nu} u_{2}$. Then the facts $l u_{1}=l u_{2}=1$ and $u_{1} / l\left(T_{\nu} u_{1}\right)=u_{2} / l\left(T_{\nu} u_{2}\right)$ imply $u_{1}=u_{2}$ so that $E_{\nu}$ is injective. Let $v$ be any function in ex. $H P_{1}\left(\Omega_{\nu} ; \partial \Omega_{\nu}\right)$. Then we have $E\left(T_{\nu}^{-1} v / l\left(T_{\nu}^{-1} v\right)\right)=v$. Assume $T_{\nu}^{-1} v / l\left(T_{\nu}^{-1} v\right)=t u_{1}+(1-t) u_{2}$ for $u_{1}, u_{2}$ in $H P_{1}\left(W_{\nu} ; \partial W_{\nu}\right)$ and a constant $t$ in $(0,1)$. Then we have $v / l\left(T_{\nu}^{-1} v\right)=t T_{\nu} u_{1}+(1-t) T_{\nu} u_{2}$ and hence $v=T_{\nu} u_{1} / l\left(T_{\nu} u_{1}\right)$ $=T_{\nu} u_{2} / l\left(T_{\nu} u_{2}\right)$. Therefore we have $T_{\nu}^{-1} v=u_{1} / l\left(T_{\nu} u_{1}\right)=u_{2} / l\left(T_{\nu} u_{2}\right)$. Since $l u_{1}=$ $l u_{2}=1$, we obtain $T_{\nu}^{-1} v / l\left(T_{\nu}^{-1} v\right)=u_{1}=u_{2}$ so that $T_{\nu}^{-1} v / l\left(T_{\nu}^{-1} v\right)$ is extreme. Then $E_{\nu}$ is also surjective. Thus we conclude

$$
\left\{\begin{array}{l}
\operatorname{dim}\left\{\bar{S}_{n}\right\}=\#\left(\operatorname{ex.} H P_{1}\left(\Omega_{1} ; \partial \Omega_{1}\right)\right)=1, \\
\operatorname{dim}\left\{\bar{S}_{n j}\right\}=\#\left(\operatorname{ex} . H P_{1}\left(\Omega_{2} ; \partial \Omega_{2}\right)\right)=2 .
\end{array}\right.
$$

\section{Firmly associated densities.}

10. Consider a $\mathscr{Y}$-sequence $\left\{\bar{Y}_{n}\right\}_{1}^{\infty}$ and a density $P$ on $\Omega$ with supp $P \subset \cup_{1}^{\infty} \bar{Y}_{n}$. Solutions $u$ of (1) on $\Omega$ are harmonic on $W=W\left(\left\{\bar{Y}_{n}\right\}\right)=\Omega-\cup_{1}^{\infty} \bar{Y}_{n}$. Then we define a mapping $T_{P}$ of $P P(\Omega ; \partial \Omega)$ to $H P(W ; \partial W)$ by

$$
T_{P} u=u-H_{u}^{W} \quad(u \in P P(\Omega ; \partial \Omega)) .
$$

Similar to the mappings $T_{1}$ and $T_{2}$ given in subsection 6 the mapping $T_{P}$ is order preserving, positively homogeneous, and additive. In general $T_{P}$ may or may not be injective and similarly surjective. If the mapping $T_{P}$ happens to be bijective, then the density $P$ is said to be canonically associated with the $\mathscr{Y}$-sequence $\left\{\bar{Y}_{n}\right\}$. If a density $P$ on $\Omega$ is canonically associated with a $\mathscr{Y}$-sequence $\left\{\bar{Y}_{n}\right\}$, then we have $\operatorname{dim} P=$ $\operatorname{dim}\left\{\bar{Y}_{n}\right\}$. To prove this we consider a mapping $E_{P}$ of $P P_{1}(\Omega ; \partial \Omega)$ to $H P_{1}(W ; \partial W)$ given by

$$
E_{P} u=\frac{T_{P} u}{l\left(T_{p} u\right)} \quad\left(u \in P P_{1}(\Omega ; \partial \Omega)\right) .
$$

Then similar to the mappings $E_{1}$ and $E_{2}$ given in subsection 9 the mapping $E_{P}$ is a bijective mapping of ex. $P P_{1}(\Omega ; \partial \Omega)$ to ex. $H P_{1}(W ; \partial W)$.

11. A density $Q$ on $\Omega$ with $P \leqslant Q, \operatorname{supp} Q \subset \cup_{1}^{\infty} \bar{Y}_{n}$ may or may not be canonically associated with a $\mathscr{Y}$-sequence $\left\{\bar{Y}_{n}\right\}_{1}^{\infty}$ even if a density $P$ on $\Omega$ is canonically associated with $\left\{\bar{Y}_{n}\right\}$. We can actually construct both examples but we will not mention them here. If any density $Q$ with $P \leqslant Q$, supp $Q \subset \cup_{1}^{\infty} \bar{Y}_{n}$ is always canonically associated with $\left\{\bar{Y}_{n}\right\}$ for a density $P$ with $\operatorname{supp} P \subseteq \cup_{1}^{\infty} \bar{Y}_{n}$, then we say that the density $P$ is firmly associated with the $\mathscr{Y}$-sequence $\left\{\bar{Y}_{n}\right\}$. Hence a firmly associated density with $\left\{\bar{Y}_{n}\right\}$ is a canonically associated density with $\left\{\bar{Y}_{n}\right\}$ and a density $Q$ with $P \leqslant Q, \operatorname{supp} Q \subset \bigcup_{1}^{\infty} \bar{Y}_{n}$ is also firmly associated with $\left\{\bar{Y}_{n}\right\}$ if a density $P$ is firmly associated with $\left\{\bar{Y}_{n}\right\}$. Now we prove the following

THEOREM 2. There always exists a density $P$ on $\Omega$ firmly associated with an arbitrarily given Yy-sequence.

The proof of this theorem will be given in subsections 15-18 after establishing auxiliary results in subsections $12-14$. 
12. Let $D$ be a Jordan region in $\Omega$. We denote by $P_{f}^{D}$ the solution of (1) on $D$ with boundary values $f$ on $\partial D$ for a density $P$ on $\Omega$ and a continuous function $f$ on $\partial D$. Let $\left\{P_{n}\right\}_{1}^{\infty}$ be a sequence of densities $P_{n}$ on $\Omega$ with

$$
\lim _{n \rightarrow \infty}\left(\inf _{D} P_{n}\right)=+\infty
$$

Then we have the following

Lemma 3. The sequence $\left\{\left(P_{n}\right)_{1}^{D}\right\}_{1}^{\infty}$ converges to zero as $n \rightarrow \infty$ uniformly on every compact subset of $D$.

For a proof we take any disk $U=U(p, r)$ in $D$ with a center $p$ and a radius $r$ and set $c_{n}=\inf _{k \geqslant n}\left(\inf _{D} P_{k}\right), w_{n}=\left(c_{n}\right)_{1}^{U}$. Since $w_{n} \geqslant w_{n+1}>0$, the sequence $\left\{w_{n}\right\}_{1}^{\infty}$ converges to a nonnegative function $w$ on $U$. Assume that $w>0$ on a subset of $U$ with positive measure. Then there exist a positive number $\delta$ and a compact subset $E$ of $\{z \in U ; w(z)>\delta\}$ such that the measure $|E|$ of $E$ is positive. If we denote the harmonic Green's function on $U$ by $g_{U}(\cdot, \cdot)$, then we have

$$
1=w_{n}(z)+\frac{1}{2 \pi} \iint_{U} g_{U}(z, \zeta) c_{n} w_{n}(\zeta) d \xi d \eta \quad(\zeta=\xi+i \eta)
$$

for any $z$ in $U$. We fix a point $q$ in $U$ and set $\sigma=\delta \inf _{E} g_{U}(q, \cdot)$. Then we have

$$
\begin{aligned}
1 & \geqslant w_{n}(q)+\frac{1}{2 \pi} \iint_{E} g_{U}(q, \zeta) c_{n} w_{n}(\zeta) d \xi d \eta \\
& \geqslant w_{n}(q)+\frac{\sigma|E| c_{n}}{2 \pi}
\end{aligned}
$$

so that

$$
|E| \leqslant \frac{2 \pi\left(1-w_{n}(q)\right)}{\sigma c_{n}}
$$

is valid for every $n=1,2, \ldots$. This contradicts the fact that $|E|>0$. Therefore $w=0$ almost everywhere on $U$. On the other hand, $w_{n}$ is rotation free:

$$
w_{n}(z)=w_{n}(|z-p|+p) \text {. }
$$

Further, by the maximum principle, $w_{n}\left(z_{1}\right) \leqslant w_{n}\left(z_{2}\right)$ if $\left|z_{1}-p\right| \leqslant\left|z_{2}-p\right|$. Then $w=0$ everywhere on $U$. Thus, by the Dini theorem, $\left\{w_{n}\right\}$ converges to zero uniformly on every compact subset of $U$ and hence we obtain Lemma 3 since $\left(P_{n}\right)_{1}^{D} \leqslant\left(c_{n}\right)_{1}^{D} \leqslant w_{n}$.

13. Let $U, V$ be Jordan regions in $\Omega$ with $\bar{U} \subset V$. Let $\left\{P_{n}\right\}_{1}^{\infty}$ be a sequence of densities $P_{n}$ on $\Omega$ such that supp $P_{n} \subset U$ and $\lim _{n}\left(\inf _{K} P_{n}\right)=+\infty$ for any compact subset $K$ of $U$. Consider a subharmonic function

$$
w(z ; U, V)= \begin{cases}\omega(\partial V ; V-\bar{U}, z) & (z \in V-\bar{U}), \\ 0 & (z \in \bar{U})\end{cases}
$$

on $V$. If we set $\|\phi\|_{E}=\sup _{E}|\phi|$ for a function $\phi$ on a subset $E$ of $\Omega$, then we have the following

LEMMA 4. $\lim _{n}\left\|\left(P_{n}\right)_{1}^{V}-w(\cdot ; U, V)\right\|_{\bar{V}}=0$. 
Since we have $\left(P_{n}\right)_{1}^{V} \leqslant\left(P_{n}\right)_{1}^{D}$ for any Jordan region $D$ in $U$ with $\bar{D} \subset U$, the sequence $\left\{\left(P_{n}\right)_{1}^{V}\right\}_{1}^{\infty}$ converges to zero uniformly on every compact subset of $U$ by Lemma 3. We take an exhaustion $\left\{U_{m}\right\}_{1}^{\infty}$ of $U$ consisting of Jordan regions $U_{m}$ in $U$ with $\bar{U}_{m} \subset U$. Set $s_{n m}=\sup _{\bar{U}_{m}}\left(P_{n}\right)_{1}^{V}(n=1,2, \ldots ; m=1,2, \ldots)$. Then $\lim _{n} s_{n m}=0$, $\left(P_{n}\right)_{1}^{V} \leqslant s_{n m}+w\left(\cdot ; U_{m}, V\right)$. Therefore we have

$$
0 \leqslant\left(P_{n}\right)_{1}^{V}-w(\cdot ; U, V) \leqslant s_{n m}+\left(w\left(\cdot ; U_{m}, V\right)-w(\cdot ; U, V)\right)
$$

on $V$. We also set $t_{m}=\sup _{\partial U} w\left(\cdot ; U_{m}, V\right)(m=1,2, \ldots)$. Then $\lim _{m} t_{m}=0$ so that we obtain Lemma 4 by the inequality

$$
\left\|\left(P_{n}\right)_{1}^{V}-w(\cdot ; U, V)\right\|_{\bar{\nu}} \leqslant s_{n m}+t_{m} .
$$

14. Let $X, Y$ be Jordan regions in $\Omega$ with $\bar{Y} \subset X$. Then we have the following simple but useful fact:

Lemma 5. For any positive number $\varepsilon$ there exists a density $P$ on $\Omega$ such that supp $P \subset Y$ and

$$
\left\|P_{f}^{X}\right\|_{\bar{Y}} \leqslant \varepsilon\|f\|_{\partial X}
$$

for any $f$ in $C(\partial X)$.

We can take a sequence $\left\{P_{n}\right\}_{1}^{\infty}$ of densities $P_{n}$ on $\Omega$ with $\operatorname{supp} P_{n} \subset Y$ and $\lim _{n}\left(\inf _{K} P_{n}\right)=+\infty$ for any compact subset $K$ of $Y$. For example, for an exhaustion $\left\{Y_{n}\right\}_{1}^{\infty}$ of $Y$ we consider nonnegative $C^{\infty}$-functions $P_{n}$ with $P_{n}=n$ on $Y_{n}$, $P_{n}=0$ on $\bar{\Omega}-Y_{n+1}$. Then by Lemma 4 we have

$$
\lim _{n \rightarrow \infty}\left\|\left(P_{n}\right)_{1}^{X}-w(\cdot ; Y, X)\right\|_{\bar{X}}=0
$$

so that $\lim _{n}\left\|\left(P_{n}\right)_{1}^{X}\right\|_{\bar{Y}}=0$. Observe that

$$
\left|\left(P_{n}\right)_{f}^{X}\right| \leqslant\left(P_{n}\right)_{|f|}^{X} \leqslant\left(P_{n}\right)_{\|f\|_{\partial X} X}^{X}=\|f\|_{\partial X}\left(P_{n}\right)_{1}^{X} .
$$

Then we have

$$
\left\|\left(P_{n}\right)_{f}^{X}\right\|_{\bar{Y}} \leqslant\left\|\left(P_{n}\right)_{1}^{X}\right\|_{\bar{Y}}\|f\|_{\partial X} .
$$

Therefore if we set $P=P_{n}$ for $n$ with $\left\|\left(P_{n}\right)_{1}^{X}\right\|_{\bar{Y}}<\varepsilon$, we obtain Lemma 5 .

15. We proceed to the proof of Theorem 2 , i.e. the existence of firmly associated densities. Let $\left\{\bar{Y}_{n}\right\}_{1}^{\infty}$ be any $\mathscr{Y}$-sequence in $\Omega$ and $X_{n}$ be a slightly larger Jordan region in $\Omega$ than $Y_{n}$ containing $\bar{Y}_{n}$. We may assume $\bar{X}_{n} \cap \bar{X}_{m}=\varnothing$ for $n \neq m$. We fix a point $z_{0}$ in $\Omega-\bigcup_{1}^{\infty} \bar{X}_{n}$ and denote by $F$ the set of nonnegative harmonic functions $u$ on $W=W\left(\left\{\bar{Y}_{n}\right\}\right)=\Omega-\bigcup_{1}^{\infty} \bar{Y}_{n}$ with $u\left(z_{0}\right)=1$. Then the Harnack inequality yields

$$
\beta_{n} \equiv \sup _{F} \max _{\partial X_{n}} u<+\infty \quad(n=1,2, \ldots) .
$$

We define a density $P=P_{W}$ on $\Omega$ by

$$
P(z)= \begin{cases}P_{n}(z) & \left(z \in \bar{X}_{n}\right), n=1,2, \ldots, \\ 0 & \left(z \in \bar{\Omega}-\bigcup_{1}^{\infty} \bar{X}_{n}\right),\end{cases}
$$

where $P_{n}$ is a density on $\Omega$ which satisfies Lemma 5 for $\varepsilon=\left(2 \beta_{n}\right)^{-1}, Y=Y_{n}$, $X=X_{n}$. We will show that $P$ is firmly associated with $\left\{\bar{Y}_{n}\right\}$. 
16. First we prove that the mapping $T_{P}$ of $P P(\Omega ; \partial \Omega)$ to $H P(W ; \partial W)$ is injective. Let $u_{1}, u_{2}$ be any functions in $P P(\Omega ; \partial \Omega)$. We remark that $u_{1}, u_{2}$ are harmonic on $W$. Then $u_{1}, u_{2}$ satisfy $u_{1} \leqslant \beta_{n} u_{1}\left(z_{0}\right), u_{2} \leqslant \beta_{n} u_{2}\left(z_{0}\right)$ on $\partial X_{n}$. Therefore by Lemma 5 we have $u_{1} \leqslant u_{1}\left(z_{0}\right) / 2, u_{2} \leqslant u_{2}\left(z_{0}\right) / 2$ on $\bigcup_{1}^{\infty} \bar{Y}_{n}$ so that $u_{1}, u_{2}$ are bounded on $\partial W=\partial \Omega \cup\left(\cup_{1}^{\infty} \partial \bar{Y}_{n}\right)$. Assume $T_{P} u_{1}=T_{P} u_{2}$. Since $u_{1}-u_{2}=H_{u_{1}-u_{2}}^{W}$ on $W, u_{1}-u_{2}$ is bounded on $W$ and hence on $\Omega$. Further $u_{1}-u_{2}$ vanishes on $\partial \Omega$. Then we have $u_{1}-u_{2} \equiv 0$.

17. Next we prove that $T_{P}$ is surjective. We set

$$
F_{X}=\bigcup_{1}^{\infty} \partial X_{n}, \quad \Gamma_{Y}=\bigcup_{1}^{\infty} \partial Y_{n} .
$$

Consider a bounded operator $K_{P}$ of $C\left(F_{x}\right)$ to $C\left(\Gamma_{Y}\right)$ defined by

$$
K_{P} \phi(z)=P_{\phi}^{X_{n}}(z) \quad\left(\phi \in C\left(F_{X}\right), z \in \partial Y_{n} ; n=1,2, \ldots\right) .
$$

Also consider a bounded operator $L_{P}$ of $C B\left(\Gamma_{Y}\right)$ to $C B\left(F_{X}\right)$ defined by

$$
L_{P} \psi(z)=H_{\psi}^{W}(z) \quad\left(\psi \in C B\left(\Gamma_{Y}\right), z \in F_{X}\right),
$$

where we set $\psi=0$ on $\partial \Omega$. Let $v$ be any function in $H P(W ; \partial W)$. Then we can construct a function $u$ in $P P(\Omega ; \partial \Omega)$ with $T_{P} u=v$ by using the bounded operator $M_{P}=L_{P} \circ K_{P}$ of a subspace of $C\left(F_{X}\right)$ to $C B\left(F_{X}\right)$. Since $v$ is dominated by the constant $\beta_{n} v\left(z_{0}\right)$ on $\partial X_{n}$, we have $K_{P}^{m} v \leqslant v\left(z_{0}\right) / 2$ on $\Gamma_{Y}$ by Lemma 5 . Then a harmonic function $M_{P} v$ is dominated by $v\left(z_{0}\right) / 2^{m}(m=1,2, \ldots)$. Therefore there exists a nonnegative function

$$
\phi_{v}=\sum_{m=0}^{\infty} M_{P}^{m} v
$$

on $F_{X}$ with $\left(I-M_{P}\right) \phi_{v}=v$, where $I$ is the identity operator of $C\left(F_{X}\right)$. Observe that $K_{P} \phi_{v}$ is a solution of (1) on $\bigcup_{1}^{\infty} X_{n}$ and $v+M_{P} \phi_{v}$ is a solution of (1) on $W$ with the same values as that of $K_{P} \phi_{v}$ on $\partial\left(W \cap\left(\cup_{1}^{\infty} X_{n}\right)\right)=F_{X} \cup \Gamma_{Y}$. Then we can define the function

$$
u(z)= \begin{cases}K_{P} \phi_{v}(z) & \left(z \in \bigcup_{1}^{\infty} X_{n}\right) \\ v(z)+M_{P} \phi_{v}(z) & (z \in W)\end{cases}
$$

in $P P(\Omega ; \partial \Omega)$ which satisfies

$$
T_{P} u=u-H_{u}^{W}=v+M_{P} \phi_{v}-L_{P}\left(K_{p} \phi_{v}\right)=v
$$

on $W$.

18. In the preceding subsections we have shown that $P$ is canonically associated with $\left\{\bar{Y}_{n}\right\}$. We turn to the proof that $P$ is firmly associated with $\left\{\bar{Y}_{n}\right\}$. Take any density $Q$ on $\Omega$ with $\operatorname{supp} Q \subset \bigcup_{1}^{\infty} \bar{Y}_{n}$ and $P \leqslant Q$. Consider densities $P_{n}, Q_{n}(n=$ $1,2, \ldots)$ on $\Omega$ defined by

$$
P_{n}(z)=\left\{\begin{array}{ll}
P(z) & \left(z \in \bar{X}_{n}\right), \\
0 & \left(z \in \bar{\Omega}-\bar{X}_{n}\right),
\end{array} \quad Q_{n}(z)= \begin{cases}Q(z) & \left(z \in \bar{X}_{n}\right), \\
0 & \left(z \in \bar{\Omega}-\bar{X}_{n}\right) .\end{cases}\right.
$$


Then $\operatorname{supp} P_{n} \subset \bar{Y}_{n}, \operatorname{supp} Q_{n} \subset \bar{Y}_{n}$ and $P_{n} \leqslant Q_{n}$. Similar to the density $P_{n}$, the density $Q_{n}$ satisfies Lemma 5 for $\varepsilon=\left(2 \beta_{n}\right)^{-1}, X=X_{n}, Y=Y_{n}$ since $\left(Q_{n}\right)_{1}^{X_{n}} \leqslant$ $\left(P_{n}\right)_{1}^{X_{n}}$. Then $Q$ is canonically associated with $\left\{\bar{Y}_{n}\right\}$ and hence $P$ is firmly associated with $\left\{\bar{Y}_{n}\right\}$.

\section{The proof of the main theorem.}

19. Let $\left\{\bar{S}_{n}\right\}_{1}^{\infty},\left\{\bar{S}_{n j}\right\}_{j=1,2 ; n \geqslant 1}$ be $\mathscr{Y}$-sequences given in subsection 6 . We can take firmly associated densities $P_{1}$ and $Q$ on $\Omega$ with $\left\{\bar{S}_{n}\right\}$ and $\left\{\bar{S}_{n j}\right\}$, respectively. We set $P=P_{1}+Q$. Then $\operatorname{supp} P \subset\left(\cup_{1}^{\infty} \bar{S}_{n}\right) \cup\left(\cup_{1}^{\infty}\left(\bar{S}_{n 1} \cup \bar{S}_{n 2}\right)\right)=\bigcup_{1}^{\infty} \bar{S}_{n}$, and $P_{1} \leqslant P$. Therefore $P$ is also canonically associated with $\left\{\bar{S}_{n}\right\}$. Thus we deduce

$$
\operatorname{dim} Q=\operatorname{dim}\left\{\bar{S}_{n j}\right\}=2, \quad \operatorname{dim} P=\operatorname{dim}\left\{\bar{S}_{n}\right\}=1,
$$

although $Q \leqslant P$.

\section{REFERENCES}

1. M. Brelot, Étude des l'équation de la chaleur $\Delta u=c(M) u(M), C(M) \geqslant 0$, au voisinage d'un point singulier du coefficient, Ann. Sci. École Norm. Sup. 48 (1931), 153-246.

2. Sur le principe des singularités positives et la notion de source pour l'équation (1) $\Delta u(M)=c(M) u(M)(c \geqslant 0)$, Ann. Univ. Lyon Sci. Math. Astro. 11 (1948), 9-19.

3. C. Constantinesc und A. Cornea, Ideale Ränder Riemannscher Flächen, Springer-Verlag, Berlin and New York, 1963.

4. M. Kawamura, On a conjecture of Nakai on Picard principle, J. Math. Soc. Japan 31 (1979), 359-371.

5. M. Kawamura and M. Nakai, A test of Picard principle for rotation free densities, II, J. Math. Soc. Japan 28 (1976), 323-342.

6. M. Nakai, A test for Picard principle, Nagoya Math. J. 56 (1974), 105-119.

7. _ A test of Picard principle for rotation free densities, J. Math. Soc. Japan 27 (1975), 412-431.

8. _ Picard principle for finite densities, Nagoya Math. J. 70 (1978), 7-24.

9. The range of Picard dimensions, Proc. Japan Acad. 55 (1979), 379-383.

10. M. Nakai and T. Tada, The distribution of Picard dimensions, Kodai Math. J. 7 (1984), 1-15.

11. R. Phelps, Lectures on Choquet's Theorem, Math. Studies No. 7, Van Nostrand, Princeton, N. J., 1965.

12. B. Rodin and L. Sario, Principal functions, Van Nostrand, Princeton, N. J., 1968.

Department of Mathematics, Nagoya Institute of Technology, Gokiso, Showa, Nagoya 466, JAPAN

Department of Mathematics, Daido Institute of Technology, Daido, Minami, Nagoya 457, JAPAN 